\title{
The major cardiovascular events of febuxostat versus allopurinol in treating gout or asymptomatic hyperuricemia: a systematic review and meta-analysis
}

\author{
Meijiao Wang ${ }^{1 \#}$, Yi Zhang ${ }^{1 "}$, Min Zhang ${ }^{1}$, Haichang Li $^{1}$, Chengping Wen ${ }^{1}$, Ting Zhao ${ }^{1}$ Zhijun Xie ${ }^{1}$, \\ Jing Sun ${ }^{2}$
}

${ }^{1}$ School of Basic Medicine Sciences, Zhejiang Chinese Medical University, Hangzhou, China; ${ }^{2}$ The Second Clinical Medical College of Zhejiang Chinese Medical University, Hangzhou, China

Contributions: (I) Conception and design: All authors; (II) Administrative support: Z Xie; (III) Provision of study materials or patients: M Wang, M Zhang; (IV) Collection and assembly of data: M Wang, Y Zhang, M Zhang; (V) Data analysis and interpretation: M Wang, M Zhang, C Wen; (VI) Manuscript writing: All authors; (VII) Final approval of manuscript: All authors.

"These authors contributed equally to this work as co-first authors.

Correspondence to: Zhijun Xie. School of Basic Medicine Sciences, Zhejiang Chinese Medical University, Hangzhou, China. Email: xzj575@163.com; Jing Sun. The Second Clinical Medical College of Zhejiang Chinese Medical University, Hangzhou, China. Email: tomorrow123go@163.com.

Backgroundk To evaluate the major cardiovascular (CV) events of febuxostat compared to allopurinol for the treatment of gout or asymptomatic hyperuricemia.

Methods: Relevant studies published until August 15, 2020 were identified by a systematic search of the PubMed and Wiley Online Library databases. Any controlled clinical trial, randomised controlled trial (RCT), retrospective cohort study or open label trial (OLT) comparing febuxostat in patients with gout or hyperuricemia with allopurinol. The quality of all identified studies was assessed based on Cochrane Collaboration's risk of bias tool. Odds ratios (OR) were calculated with random effects and reported with corresponding $95 \%$ confidence intervals (CI).

Results: Eighteen studies were ultimately included in the analysis, among them 6 articles mentioned serum uric acid (sUA) level before and after treatment, 14 articles mentioned major cardiovascular events, 5 articles mentioned cardiovascular death, 6 articles mentioned skin reactions, 6 articles mentioned musculoskeletal and connective tissue signs and symptoms, 4 articles mentioned joint-related signs and symptoms, 6 articles mentioned upper respiratory infection, 5 articles mentioned gastrointestinal reaction and 7 articles mentioned all-cause mortality. The febuxostat group showed significantly lower sUA levels than allopurinol group ( $\mathrm{MD}=-0.83,95 \% \mathrm{CI}:-1.22$ to $\left.-0.44, \mathrm{P}<0.0001, \mathrm{I}^{2}=98 \%\right)$. There was no markedly difference between the febuxostat and allopurinol (OR $1.01,95 \% \mathrm{CI}: 0.83$ to $1.23, \mathrm{P}=0.84, \mathrm{I}^{2}=95 \%$ ) in the major cardiovascular events. The occurrence of skin reactions of febuxostat was significantly fewer than allopurinol (OR 0.55, 95\% CI: 0.42 to $0.73, \mathrm{P}<0.0001, \mathrm{I}^{2}=49 \%$ ). Regarding to occurrence of $\mathrm{CV}$ death, musculoskeletal and connective tissue signs and symptoms, febuxostat group was higher than allopurinol group. However, among patients with gout or hyperuricemia, treatment with febuxostat resulted in other adverse reactions, including all-causes mortality similar to those associated with allopurinol.

Discussion: The limitation of the study was the included studies show high heterogeneity in regard to their design. There was no difference in the incidence of major cardiovascular events between febuxostat and allopurinol, and febuxostat was better in lowering uric acid and has less adverse skin reactions than allopurinol, but the risk of $\mathrm{CV}$ death of febuxostat was higher than allopurinol.

Keywords: Gout; hyperuricemia; febuxostat; allopurinol; major cardiovascular events; meta-analysis

Submitted Jun 11, 2021. Accepted for publication Jul 30, 2021.

doi: 10.21037/apm-21-1564

View this article at: https://dx.doi.org/10.21037/apm-21-1564 


\section{Introduction}

Gout has become a common clinical disease in the past few decades. The worldwide prevalence rate reported to be ranging from $2.6 \%$ to $36 \%$ in different populations (1). The prevalence of hyperuricemia and gout were $13.3 \%(19.4 \%$ in men and $7.9 \%$ in women) and $1.1 \%$, respectively in mainland China (2), and $21 \%(21.2 \%$ in men and $21.6 \%$ in women) and 3.9\%, respectively in the United States (3).

Hyperuricemia and gout are independent risk factors for cardiovascular disease (CVD) such as hypertension (4), myocardial infarction (MI) $(5,6)$, etc. So, urate-lowering agents might be beneficial to CVDs while lowering serum uric acid levels.

Allopurinol has been the main xanthine oxidase inhibitor used to reduce uric acid in clinic before the presence of febuxostat. However, in clinical application, there was $0.33 \%$ incidence of allopurinol hypersensitivity syndrome (7) characterized by symptoms of severe skin rash (manifesting by toxic epidermal necrolysis, erythema multiforme, diffuse maculopapular rash or exfoliative dermatitis), acute hepatocellular injury, worsening renal function and fever, etc. $(8,9)$. The allopurinol hypersensitivity syndrome is potentially life-threatening, and is associated with significant mortality (10).

So, febuxostat, a non-purine xanthine inhibitor as a better alternative to allopurinol, has become the widely used urate-lowering agent (11). However, researchers still have different views into the advantages of febuxostat versus allopurinol in reducing cardiovascular $(\mathrm{CV})$ events and other events $(12,13)$. So, we did a systematic review and meta-analysis comparing the safety (especially cardiovascular events) and efficacy of febuxostat compared to allopurinol in the treatment of gout or hyperuricemia.

\section{Methods}

\section{Data sources and search strategy}

This systematic review followed the Preferred Reporting Items for Systematic Reviews and Meta-Analyses (PRISMA) statement (available at https://dx.doi.org/10.21037/apm21-1564). The protocol of this study was registered and approved by PROSPERO under the registration number of CRD42021206233.

Relevant studies published until August 15, 2020 were identified by a systematic search of the PubMed and Wiley Online Library databases, using the following key words: (((Febuxostat OR TEI 6720 OR 6720, TEI OR TEI-6720
OR TEI6720 OR loric)) AND ((Allopurinol OR Uribenz OR allopurinol OR allori OR all argon OR allura OR Pan Quimica OR Apulonga OR azurin OR Atisuril OR Bleminol OR caplan al OR caprate OR Cellidrin OR Embarin OR suspends OR coligan OR hamartin OR Lopurin OR Lysuron OR Lysuron OR jena purinyl OR Milurit OR Milurite OR novopro OR Uripurinol OR ursin OR urtis OR xanthomas OR ureidoacid OR xanthoria OR zygote OR Zyloprim OR pyloric OR pure duct OR purinyl OR Progout OR remind OR rima purinyl OR rouco OR Tipuric OR aldohexa OR al lohedan OR allo prin))) in combination with 'Gout or hyperuricemia' or (((febuxostat) and allopurinol) and (gout or hyperuricemia). In addition, the reference lists of eligible studies were screened by Clinical Trial to identify potential relevant studies. Unpublished data were not included in the study. Two authors reviewed the articles independently, disputes were resolved by discussion or by consultation of a third investigator for potential inclusion in the present metaanalysis.

\section{Inclusion criteria}

Any controlled clinical trial, randomised controlled trial (RCT), retrospective cohort study or open label trial (OLT) comparing febuxostat (alone or combined with any other drugs) in patients with gout or hyperuricemia with allopurinol (alone or combined with any other drugs). Any dosage regimen, follow-up duration and sample size were allowed. Patients at least 18 years meeting the preliminary American College of Rheumatology (ACR) criteria for gout or given a diagnosis of gout or hyperuricemia as described by the authors. We excluded reviews, cross sectional studies and case reports.

\section{Data extraction}

Data extraction was carried out independently by two authors (M Wang and M Zhang) using standard data extraction forms, and disputes were resolved by discussion or by consultation of a third investigator (Y Zhang). Only outcomes related to safety were incorporated into the analysis. The selected manuscripts were reviewed in further detail, and data were extracted using standard forms. The following information were extracted from each study: first author, year of publication, sample size, mean age, male rate, treatment in the intervention and control groups and co-morbid conditions (diabetes, hyperlipidaemia, $\mathrm{CV}$ diseases and hypertension). Furthermore, the study 
extracted the serum uric acid (sUA) levels prior to and after the administration of febuxostat or allopurinol, the major cardiovascular events (including HF, stroke, MI, heart failure, atrial fibrillation, cardiovascular abnormalities, etc.), $\mathrm{CV}$ death, all-cause mortality, and other adverse reactions (skin reactions, musculoskeletal and connective tissue signs and symptoms, joint-related signs and symptoms, upper respiratory infection (URI), gastrointestinal reaction). When raw data were not provided, data were extracted from figures and tables to calculate the necessary information.

\section{Quality assessment}

The quality of all identified studies was assessed based on Cochrane Collaboration's risk of bias tool. It assesses for random sequence generation, allocation concealment, blinding of participants and personnel, blinding of outcome assessment, incomplete out-come data, selective reporting and other sources of bias. Each item was evaluated as low, unclear or high risk of bias with supportive data from the study.

\section{Statistical analysis}

The sUA levels in patients with gout or hyperuricemia based on the mean difference (MD) with $95 \%$ confidence intervals (95\% CIs). Major CV events, CV death, all-cause mortality and adverse reactions are presented as odds ratios (OR) with 95\% CIs (14). We explored the effect of the dosage of febuxostat by subset analyses. We employed fixedeffect models in the studies with low heterogeneity defined as an $\mathrm{I}^{2}$ value less than $50 \%$, and random effect models in the studies with substantial heterogeneity defined as an $\mathrm{I}^{2}$ value more than $50 \%$. We used two-tailed $\mathrm{P}$ values, $\mathrm{P}<0.05$ was considered significant. We performed the statistical analyses with Review Manager (RevMan), Version 5.3 (15).

\section{Subgroup and sensitivity analyses}

We evaluated subgroups according to the febuxostat dose were 40 and $\geq 80 \mathrm{mg}$. Sensitivity described was performed using a one-by-one cull of the literature.

\section{Results}

\section{Search results}

We identified 790 citations after preliminary screening, including 540 from PubMed and 250 from Wiley Online Library. The flow diagram and outcomes of the study are shown in Figure S1. We selected 48 studies for full-text review and excluded 30 non-conforming experiments, and finally obtained 18 literatures (11-13,16-30). Among them, 6 articles mentioned sUA level before and after treatment (16,20-24), 14 articles mentioned the major cardiovascular events in the end points (11-13,17-21,24,26-30), 5 articles mentioned $\mathrm{CV}$ death $(12,19,27,28,30), 7$ articles mentioned all-cause mortality (11-13,18,27-29), 6 articles mentioned skin reactions $(16,18,19,24,25,28), 6$ articles mentioned musculoskeletal and connective tissue signs and symptoms (16-19,21,24), 4 articles mentioned joint-related signs and symptoms (16-18,21), 6 articles mentioned URI (17-21,24), and 5 articles mentioned gastrointestinal reaction $(16-18,21,24)$.

\section{Description of the included studies and participants}

The characteristics of included studies were summarized in Table 1. All studies recruited patients with a diagnosis of gout or hyperuricemia. A total of 278,039 patients were included. Nine trials were based in North America with 120,088 patients $(43.19 \%)(11,12,16-19,21,26,30)$, eight were from Asia with a recruitment of 157,934 participants (56.80\%) (13,20,23-25,27-29), one was European studies with 17 patients $(0.001 \%)$ (22). The range of mean age was $45-76$ years. Men accounted for 52.3-97.9\% . 5-55.15\% patients combined diabetes, $2.5-82.9 \%$ combined hyperlipidemia, $0.3-92.9 \%$ combined CVD, and $15.5-$ 95.4\% combined hypertension.

\section{Quality assessment}

The risk of bias for each study was summarized in Figure S2. There was identified 10 relevant RCTs (12,16-19,21,2325,30), 6 retrospective cohort study (11,13,26-29), 1 open prospective non-randomized study (22), 1 doubleblind comparative study (20). There were about half trials described random sequence generation and allocation concealment. We evaluated blinding of participants and personnel (performance bias) and blinding of outcome assessment (detection bias). Eight trials were deemed to have a risk of bias given a lack of blinding.

\section{Endpoints}

There were six studies mentioned sUA levels before and 


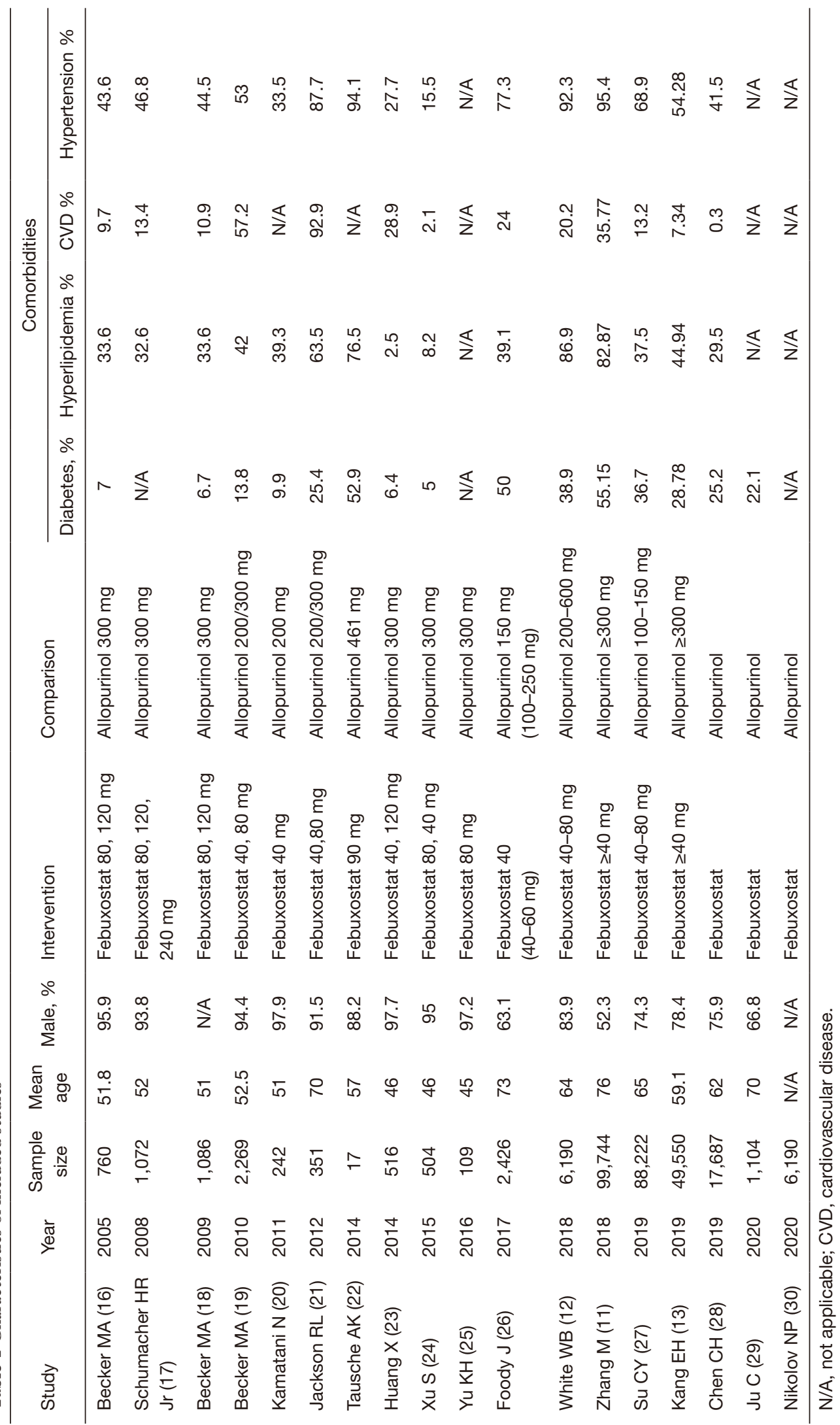




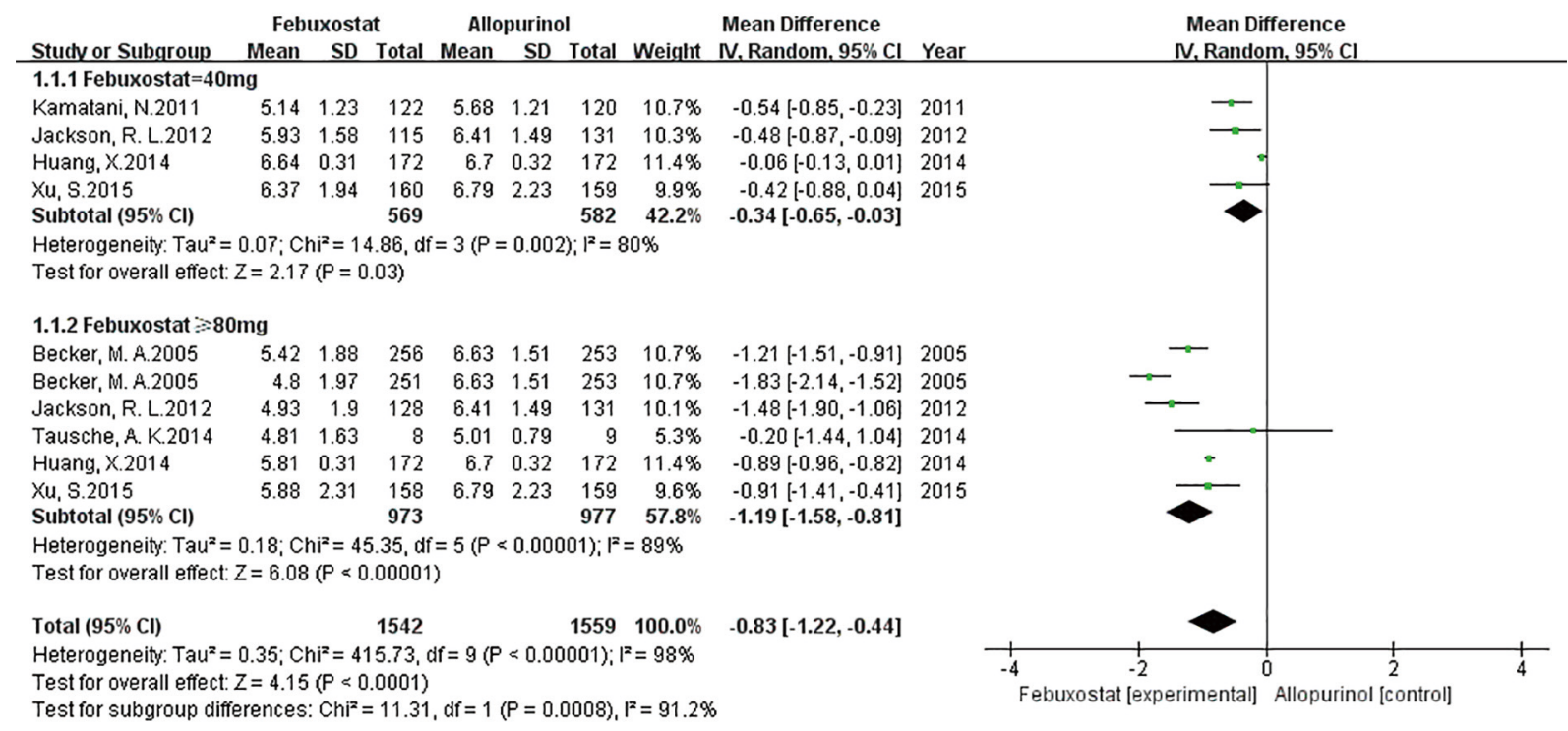

Figure 1 sUA level of febuxostat and allopurinol in gout or hyperuricemia. sUA, serum uric acid.

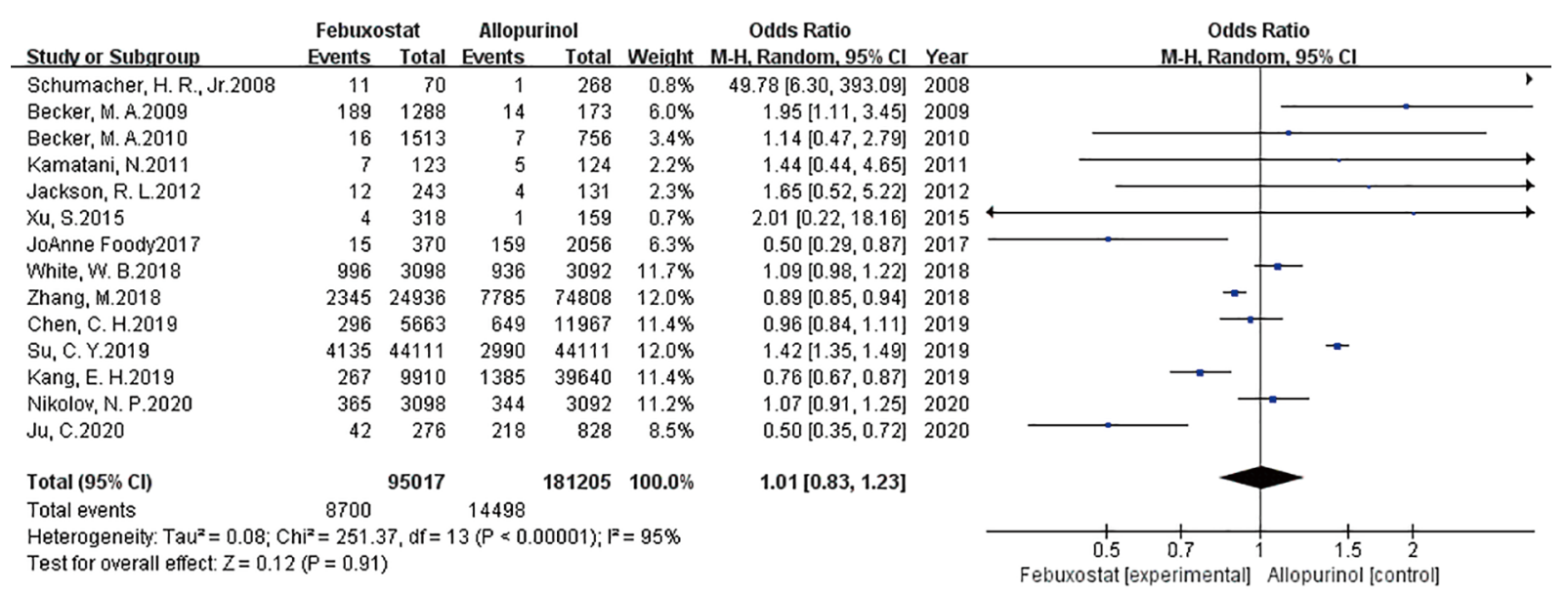

Figure 2 Odds ratios of major cardiovascular events of febuxostat and allopurinol in gout or hyperuricemia.

after treatment. Compared with the allopurinol group, the febuxostat group shown significantly lower sUA levels in the overall study population ( $\mathrm{MD}=-0.83,95 \% \mathrm{CI}:-1.22$ to $-0.44, \mathrm{P}<0.0001$, Figure 1). A subgroup analyses on different dosages of febuxostat was performed. In each subgroup, febuxostat group showed significantly lower mean sUA levels than that of allopurinol group ( $\mathrm{MD}=-0.34,95 \% \mathrm{CI}$ : -0.65 to $-0.03, P=0.03$ for febuxostat $=40 \mathrm{mg} ; \mathrm{MD}=-1.19$, $95 \%$ CI: -1.58 to $-0.81, \mathrm{P}<0.00001$ for febuxostat $\geq 80 \mathrm{mg}$, Figure 1). The uric acid lowering effect in febuxostat $\geq 80 \mathrm{mg}$ was better than that in febuxostat $=40 \mathrm{mg}(\mathrm{P}=0.0008)$.

In evaluating major cardiovascular events in gout or hyperuricemia, fourteen studies compared febuxostat ( $\mathrm{n}=95,017$ patients) vs. allopurinol ( $\mathrm{n}=181,205$ patients) (Figure 2). Overall, the pooled analysis shown that there was no significant difference between the two groups (febuxostat vs. allopurinol: OR $1.01,95 \%$ CI: 0.83 to $1.23, \mathrm{P}=0.91$ ).

The occurrence of skin reactions of febuxostat was significantly fewer than that of allopurinol (OR 0.55, 95\% CI: 0.42 to $0.73, \mathrm{P}<0.0001$, Figure $3 A$ ). Regarding to the 


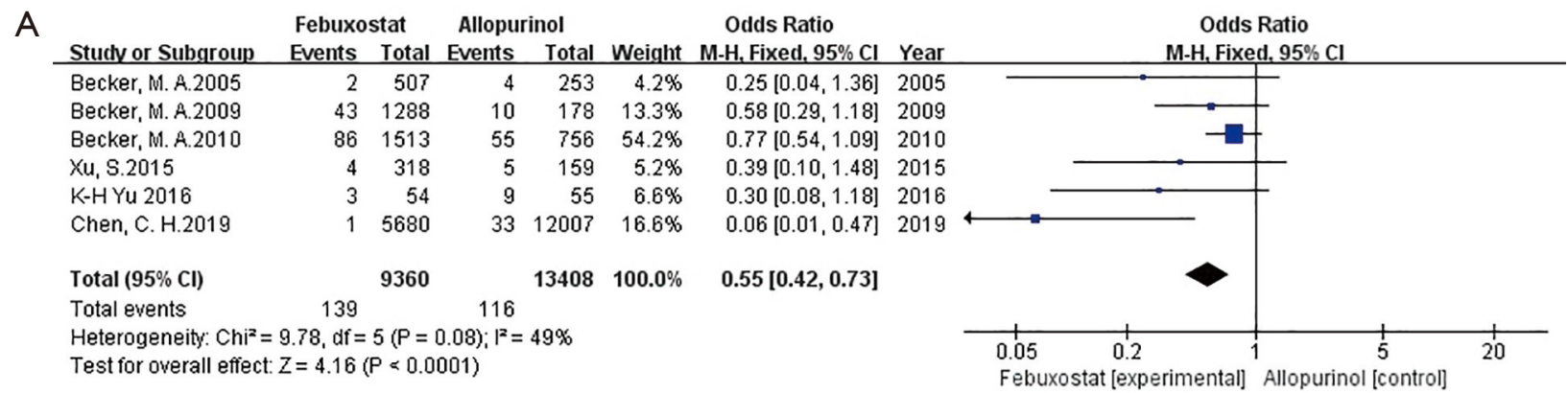

B

\begin{tabular}{|c|c|c|c|c|c|c|c|c|c|c|}
\hline \multirow[b]{2}{*}{ Study or Subgroup } & \multicolumn{2}{|c|}{ Febuxostat } & \multicolumn{2}{|c|}{ Allopurinol } & \multicolumn{3}{|c|}{ Odds Ratio } & \multirow{2}{*}{\multicolumn{3}{|c|}{$\begin{array}{c}\text { Odds Ratio } \\
\text { M.H. Fixed. } 95 \% \mathrm{Cl}\end{array}$}} \\
\hline & Events & Total & Events & Total & Weight & M-H, Fixed, $95 \% \mathrm{Cl}$ & Year & & & \\
\hline Becker, M. A.2010 & 0 & 1513 & 2 & 756 & $0.6 \%$ & $0.10[0.00,2.08]$ & 2010 & & & \\
\hline White, W. B.2018 & 134 & 3098 & 100 & 3092 & $18.1 \%$ & $1.35[1.04,1.76]$ & 2018 & & & \\
\hline Su, C. Y.2019 & 468 & 44111 & 334 & 44111 & $62.4 \%$ & $1.41[1.22,1.62]$ & 2019 & & & \\
\hline Chen, C. H.2019 & 5 & 5663 & 7 & $1196 ?$ & $0.8 \%$ & $1.51[0.48,4.76]$ & 2019 & & & \\
\hline Nikolov, N. P.2020 & 134 & 3098 & 100 & 3092 & $18.1 \%$ & $1.35[1.04,1.76]$ & 2020 & & & \\
\hline Total $(95 \% \mathrm{Cl})$ & & 57483 & & 63018 & $100.0 \%$ & $1.38[1.23,1.54]$ & & & & \\
\hline Total events & 741 & & 543 & & & & & & & \\
\hline $\begin{array}{l}\text { Heterogeneity: Chi } \\
\text { Test for overall effe }\end{array}$ & $\begin{array}{l}3.01, d f= \\
Z=5.64\end{array}$ & $\begin{array}{l}4(P=0 \\
P<0.00\end{array}$ & $\begin{array}{l}.56) ;\left.\right|^{2}= \\
0001)\end{array}$ & & & & & $\begin{array}{cc}0.5 & 0.7 \\
\text { Febuxostat [experimental }\end{array}$ & $\begin{array}{c}1.5 \\
\text { Allopurinol }\end{array}$ & 2 \\
\hline
\end{tabular}

C

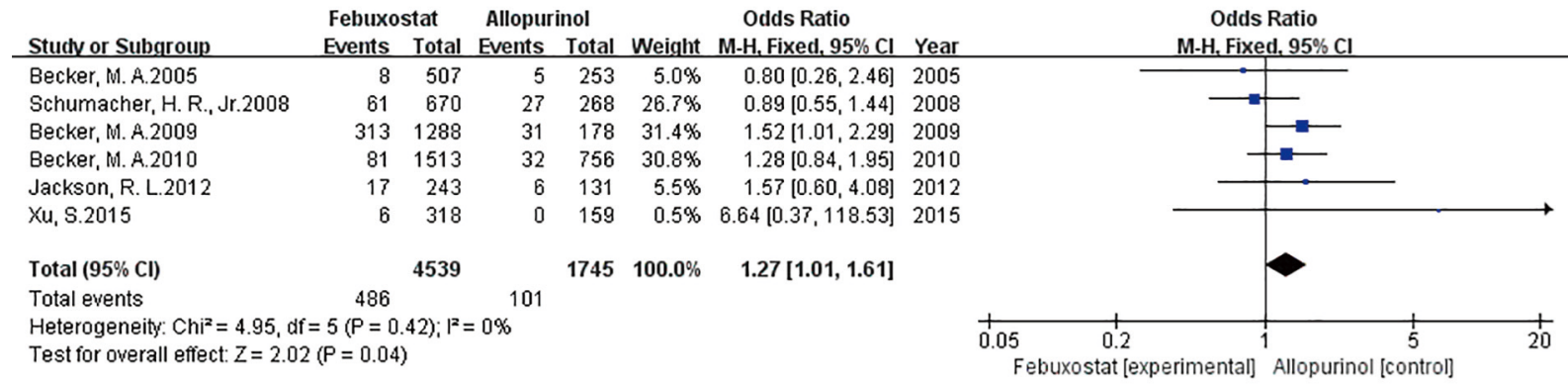

D

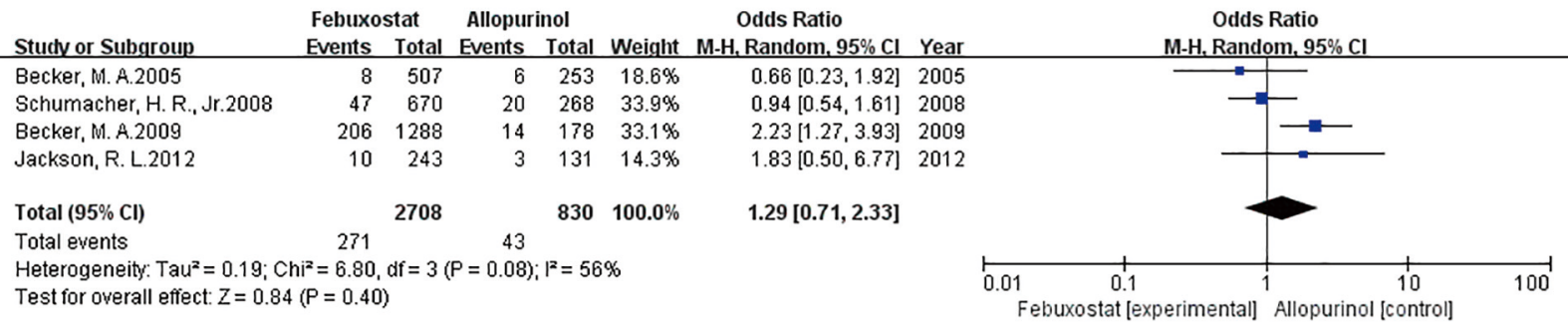

E

Febuxostat Allopurinol Odds Ratio

Odds Ratio Odds Ratio

\begin{tabular}{|c|c|c|c|c|c|c|c|c|c|c|}
\hline \multirow[b]{2}{*}{ Stucty or Subgroup } & \multicolumn{2}{|c|}{ Febuxostat } & \multicolumn{2}{|c|}{ Allopurinol } & \multicolumn{3}{|c|}{ Odds Ratio } & \multirow{2}{*}{\multicolumn{3}{|c|}{$\begin{array}{l}\text { Odds Ratio } \\
\text { M-H, Random, } 95 \% \mathrm{Cl}\end{array}$}} \\
\hline & Events & Total & Events & Total & Weight & M-H, Random, $95 \% \mathrm{Cl}$ & Year & & & \\
\hline Schumacher, H. R., Jr.2008 & 118 & 670 & 52 & 268 & $26.4 \%$ & $0.89[0.62,1.28]$ & 2008 & $\longrightarrow$ & — & \\
\hline Becker, M.A.2009 & 556 & 1288 & 38 & 178 & $26.2 \%$ & $2.80[1.92,4.07]$ & 2009 & & & \\
\hline Becker, M. A.2010 & 124 & 1513 & $5 ?$ & 756 & $27.0 \%$ & $1.09[0.79,1.52]$ & 2010 & & $=$ & \\
\hline Jackson, R. L.2012 & 20 & 243 & 9 & 131 & $17.8 \%$ & $1.22[0.54,2.75]$ & 2012 & & $\rightarrow$ & \\
\hline Xu, S.2015 & 1 & 318 & 0 & 159 & $2.6 \%$ & $1.51[0.06,37.20]$ & 2015 & & & \\
\hline Total $(95 \% \mathrm{Cl})$ & & 4032 & & 1492 & $100.0 \%$ & $1.36[0.79,2.33]$ & & & & \\
\hline Total events & 819 & & 156 & & & & & & & \\
\hline $\begin{array}{l}\text { Heterogeneity: } \operatorname{Tau}^{2}=0.25 \\
\text { Test for overall effect: } Z=1.1\end{array}$ & $\begin{array}{l}\mathrm{i}^{2}=21.42 \\
(P=0.26)\end{array}$ & 2, $d f=4$ & $(P=0.00$ & $03) ; 1^{2}=$ & $=81 \%$ & & & $\begin{array}{cc}0.2 & 0.5 \\
\text { Febuxostat [experimental] }\end{array}$ & $\frac{1}{1}$ & 5 \\
\hline
\end{tabular}




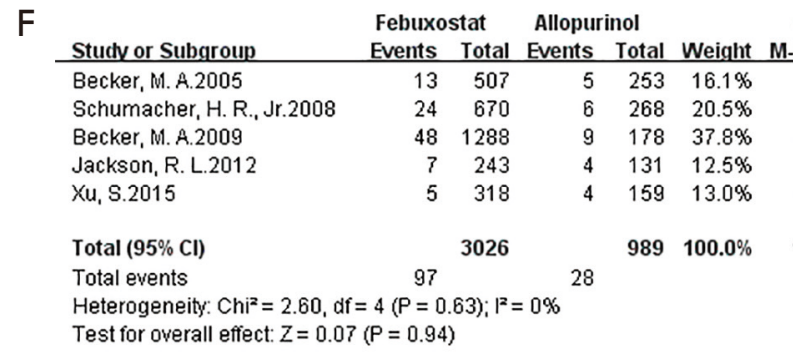

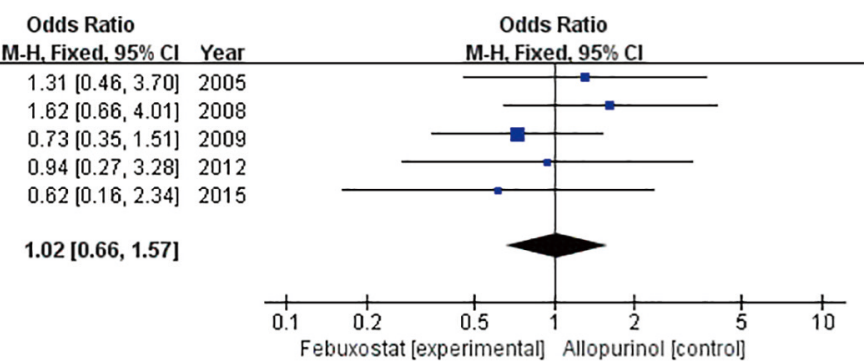

\begin{tabular}{|c|c|c|c|c|c|c|c|}
\hline \multirow[b]{2}{*}{ Study or Subgroup } & \multicolumn{2}{|c|}{ Febuxostat } & \multicolumn{2}{|c|}{ Allopurinol } & \multicolumn{3}{|c|}{ Odds Ratio } \\
\hline & Events & Total & Events & Total & Weight & M-H. Random, $95 \% \mathrm{Cl}$ & Year \\
\hline Becker, M.A.2009 & 10 & 1288 & 0 & 173 & $0.6 \%$ & $2.85[0.17,48.85]$ & \\
\hline White, W. B.2018 & 243 & 3098 & 199 & 3092 & $18.9 \%$ & $1.24[1.02,1.50]$ & \\
\hline Zhang, M.2018 & 1144 & 24936 & 4022 & 74808 & $21.6 \%$ & $0.85[0.79,0.91]$ & \\
\hline Chen, C. H.2019 & 5 & 5680 & 15 & 12007 & $4.0 \%$ & $0.70[0.26,1.94]$ & \\
\hline Kang, E. H.2019 & 135 & 9910 & 545 & 39640 & $19.0 \%$ & $0.99[0.82,1.20]$ & \\
\hline Su.C.Y.2019 & 1630 & 44111 & 1301 & 44111 & $21.5 \%$ & $1.26[1.17,1.36]$ & \\
\hline Ju, C.2020 & 52 & 276 & 204 & 828 & $14.5 \%$ & $0.71[0.51,1.00]$ & \\
\hline Total $(95 \% \mathrm{CI})$ & & 89299 & & 174659 & $100.0 \%$ & $1.00[0.80,1.24]$ & \\
\hline $\begin{array}{l}\text { Total events } \\
\text { Heterogeneity: Tau } \\
\text { Test for overall effer }\end{array}$ & $\begin{array}{l}3219 \\
0.06 ; \mathrm{Chi} \\
=0.04\end{array}$ & $\begin{array}{l}2=70.6 \\
P=0.9\end{array}$ & $\begin{array}{c}6286 \\
d f=6(\end{array}$ & $<0.00$ & 01); $\left.\right|^{2}=$ & & \\
\hline
\end{tabular}

Figure 3 Relative risks of adverse events of febuxostat and allopurinol in gout or hyperuricemia. (A) Skin reactions; (B) CV death: cardiovascular death; (C) musculoskeletal and connective tissue signs and symptoms; (D) joint-related signs and symptoms; (E) URI: upper respiratory infection; (F) gastrointestinal reaction; (G) all-cause mortality.

risks of $\mathrm{CV}$ death and musculoskeletal and connective tissue signs and symptoms, febuxostat were higher than allopurinol (OR 1.38, 95\% CI: 1.23 to $1.54, \mathrm{P}<0.00001$ for $\mathrm{CV}$ death, Figure 3B; OR 1.27, 95\% CI: 1.01 to 1.61 , $\mathrm{P}=0.04$ for musculoskeletal and connective tissue signs and symptoms Figure 3C). As to other adverse reactions, treatment with febuxostat similar to those associated with allopurinol (Figure 3D, joint-related signs and symptoms, OR $1.29,95 \%$ CI: 0.71 to $2.33, \mathrm{P}=0.40$, Figure $3 E$, URI OR $1.36,95 \%$ CI: 0.79 to $2.33, \mathrm{P}=0.26$, Figure $3 F$ gastrointestinal reaction OR 1.02, 95\% CI: 0.66 to 1.57 , $\mathrm{P}=0.94$, Figure $3 G$ all-cause mortality OR $1.00,95 \%$ CI: 0.80 to $1.24, \mathrm{P}=0.97)$.

\section{Sensitivity analysis and publication bias}

We used the MD or OR to assess the robustness of our results (14). The main results did not change in the sensitivity analyses of major cardiovascular events and sUA levels in all studies (Tables 2,3). The MD of sUA levels and OR of major CV events were not significantly changed, indicating that the results were stable.

Some asymmetry was noted in the comparison of major $\mathrm{CV}$ events. However, no further testing was performed for the limited number of studies. Funnel plots of the major cardiovascular events, sUA levels and the other adverse events comparing between febuxostat and allopurinol shown in Figure S3.

\section{Discussion}

Allopurinol has been demonstrated to delay the time to angina onset (31) and shown to have potential preventions of MI, stroke, atrial fibrillation, and other CVDs in observational studies in select populations (32) and reported to decrease $\mathrm{CV}$ mortality and heart failure readmissions (33-35). However, not all results were concordant. In a study, allopurinol was found a moderate increase in CV risk (36). Another study didn't find any correlation between $\mathrm{CV}$ risk and initiation of xanthine oxidase inhibitors in gout patients (37). The different outcomes may be related to age of patients, comorbidities, drugs taken and follow-up time. Febuxostat has shown significantly increased risk of $\mathrm{CV}$ mortality and severe CV events compared with allopurinol $(12,27)$. However, there were studies revealed no difference in the risk of non-fatal CV events and all-cause mortality between them $(28,29)$. Due to the inconsistency of the research results, 
Table 2 Sensitivity analysis of major cardiovascular events

\begin{tabular}{lccc}
\hline & OR & $\mathrm{I}^{2} / \%$ & $\mathrm{P}$ value \\
\hline Total & $1.03(0.83,1.28)$ & 95 & 0.78 \\
Exclusion of literature & & & \\
Schumacher HR Jr 2008 & $0.99(0.80,1.22)$ & 95 & 0.93 \\
Becker MA 2009 & $0.98(0.79,1.23)$ & 95 & 0.88 \\
Becker MA 2010 & $1.03(0.83,1.28)$ & 95 & 0.81 \\
Kamatani N 2011 & $1.02(0.82,1.27)$ & 95 & 0.84 \\
Jackson RL 2012 & $1.02(0.82,1.27)$ & 95 & 0.87 \\
Xu S 2015 & $1.03(0.83,1.27)$ & 95 & 0.82 \\
Foody J 2017 & $1.09(0.87,1.36)$ & 95 & 0.45 \\
Zhang M 2018 & $1.06(0.83,1.36)$ & 91 & 0.62 \\
White WB 2018 & $1.03(0.81,1.32)$ & 95 & 0.79 \\
Chen CH 2019 & $1.11(0.89,1.38)$ & 95 & 0.35 \\
Su CY 2019 & $0.95(0.80,1.12)$ & 81 & 0.52 \\
Kang EH 2019 & $1.08(0.86,1.35)$ & 95 & 0.53 \\
Nikolov NP 2020 & $1.03(0.81,1.31)$ & 95 & 0.80 \\
\hline
\end{tabular}

OR, odds ratios.

Table 3 Sensitivity analysis of sUA levels

\begin{tabular}{lccc}
\hline & MD & $1^{2} / \%$ & $P$ value \\
\hline Total & $-0.83(-1.22,-0.44)$ & 98 & $<0.0001$ \\
Exclusion of literature & & & \\
Becker MA 2005 & $-0.64(-1.06,-0.23)$ & 98 & 0.002 \\
Kamatani N 2011 & $-0.86(-1.29,-0.44)$ & 98 & $<0.0001$ \\
Jackson RL 2012 & $-0.79(-1.23,-0.35)$ & 98 & 0.0004 \\
Huang X 2014 & $-0.94(-1.35,-0.53)$ & 88 & $<0.00001$ \\
Tausche AK 2014 & $-0.86(-1.27,-0.46)$ & 98 & $<0.0001$ \\
\hline
\end{tabular}

sUA, serum uric acid; MD, mean difference.

we conducted the large sample systematic review and shown no difference in major cardiovascular events and all-cause mortality between febuxostat and allopurinol in the treatment of gout or hyperuricemia, but CV death in febuxostat was more than allopurinol. The results were reliable with a large sample size. Strangely, all-cause mortality was neutral between drugs but the risk of $\mathrm{CV}$ death of febuxostat group was higher than allopurinol. The discordance could be cause by other lethal factors, such as the allopurinol hypersensitivity syndrome, which was associated with significant mortality. However, because of no detailed information was given in the included study, no other serious CV events were observed in this manuscript.

Elevated serum uric acid can cause endothelial dysfunction, platelet adhesiveness and production of pro-inflammatory substances, accelerating underlying CV diseases. Lowering sUA levels has been conferred to have a certain degree benefits of $\mathrm{CV}$ protection $(38,39)$. Allopurinol is cost effective and efficacious as first-line therapy for gout. Febuxostat is a $\mathrm{XO}$ inhibitor that is metabolized in the liver in contrast to allopurinol, and is usually used clinically in patients who have allopurinol-associated adverse events, or do not achieve target sUA on therapeutic doses (40). The studies demonstrated greater urate-lowering ability of therapeutic doses of febuxostat when compared with low-dose allopurinol (32) and shown better efficacy for febuxostat in reducing sUA and vascular endothelial damage (41). A systematic review investigated the number of patients that febuxostat and allopurinol achieving a target serum uric acid level of $<6 \mathrm{mg} / \mathrm{dL}$, the result shown no evidence that febuxostat was superior to allopurinol for clinically relevant outcomes (42). Febuxostat display highly selective and potent inhibition of xanthine oxidase and greater hypouricemic activity than do commonly used dosage of allopurinol, and the study showed febuxostat was better than allopurinol in reducing serum uric acid.

Allopurinol was limited in clinical by severe skin reactions, hypersensitivity, which were considered as be related to human leukocyte anti-gene B*5801 allele (43). Chen et al. studied that the overall incidence of hypersensitivity reactions of allopurinol-related was significantly higher than that of febuxostat-related (28). In the study, febuxostat resulted in a lower incidence of adverse skin reactions compared with allopurinol. The study also considered other adverse events (musculoskeletal and connective tissue signs and symptoms, joint-related signs and symptoms, URI and gastrointestinal reactions), except for higher incidence of musculoskeletal and connective tissue signs and symptoms in the febuxostat group, there were no difference in the incidence of the others.

In the present study, the included studies showed high heterogeneity in regard to their design (both randomized and observational), with different patient characteristics, dosage of febuxostat and allopurinol, treatment duration or follow-up time. For the limited number of studies and different treatment outcomes included in the present meta-analysis, it was not possible to further investigate for subgroup analysis of major cardiovascular events. There 
were not given cardiovascular events associated with gout and hyperuricemia respectively in the literatures, so a stratified analysis cannot be performed.

\section{Conclusions}

In conclusion, the meta-analysis has shown no difference in the incidence of major cardiovascular events between febuxostat and allopurinol, but febuxostat was better in lowering uric acid and has less adverse skin reactions than allopurinol. However, the risk of $\mathrm{CV}$ death of febuxostat was higher than allopurinol. In clinical, allopurinol and febuxostat should be commenced at an initial dose, the dose increased gradually and pay close attention to the major cardiovascular events, the febuxostat CV death, allopurinol hypersensitivity syndrome.

\section{Acknowledgments}

We very appreciated Yingying Mao at Zhejiang Chinese Medical University for her help with the language of this manuscript.

Funding: This work was supported by the National Natural Science Foundation of China (No.81803973).

\section{Footnote}

Reporting Checklist: The authors have completed the PRISMA reporting checklist. Available at https://dx.doi. org/10.21037/apm-21-1564

Peer Review File: Available at https://dx.doi.org/10.21037/ apm-21-1564

Conflicts of Interest: All authors have completed the ICMJE uniform disclosure form (available at https://dx.doi. org/10.21037/apm-21-1564). The authors report receiving funding from the National Natural Science Foundation of China (No. 81803973) for this work.

Ethical Statement: The authors are accountable for all aspects of the work in ensuring that questions related to the accuracy or integrity of any part of the work are appropriately investigated and resolved. The present study was registered and approved by PROSPERO under the registration number of CRD42021206233.

Open Access Statement: This is an Open Access article distributed in accordance with the Creative Commons Attribution-NonCommercial-NoDerivs 4.0 International License (CC BY-NC-ND 4.0), which permits the noncommercial replication and distribution of the article with the strict proviso that no changes or edits are made and the original work is properly cited (including links to both the formal publication through the relevant DOI and the license). See: https://creativecommons.org/licenses/by-nc-nd/4.0/.

\section{References}

1. Uaratanawong S, Suraamornkul S, Angkeaw S, et al. Prevalence of hyperuricemia in Bangkok population. Clin Rheumatol 2011;30:887-93.

2. Liu R, Han C, Wu D, et al. Prevalence of Hyperuricemia and Gout in Mainland China from 2000 to 2014: A Systematic Review and Meta-Analysis. Biomed Res Int 2015;2015:762820.

3. Zhu Y, Pandya BJ, Choi HK. Prevalence of gout and hyperuricemia in the US general population: the National Health and Nutrition Examination Survey 2007-2008. Arthritis Rheum 2011;63:3136-41.

4. van Durme C, van Echteld IA, Falzon L, et al. Cardiovascular risk factors and comorbidities in patients with hyperuricemia and/or gout: a systematic review of the literature. J Rheumatol Suppl 2014;92:9-14.

5. De Vera MA, Rahman MM, Bhole V, et al. Independent impact of gout on the risk of acute myocardial infarction among elderly women: a population-based study. Ann Rheum Dis 2010;69:1162-4.

6. Krishnan E, Baker JF, Furst DE, et al. Gout and the risk of acute myocardial infarction. Arthritis Rheum 2006;54:2688-96.

7. Jordan KM, Cameron JS, Snaith M, et al. British Society for Rheumatology and British Health Professionals in Rheumatology guideline for the management of gout. Rheumatology (Oxford) 2007;46:1372-4.

8. Singer JZ, Wallace SL. The allopurinol hypersensitivity syndrome. Unnecessary morbidity and mortality. Arthritis Rheum 1986;29:82-7.

9. Hande KR, Noone RM, Stone WJ. Severe allopurinol toxicity. Description and guidelines for prevention in patients with renal insufficiency. Am J Med 1984;76:47-56.

10. Pawar P, Gupta VA, Karnik ND, et al. Allopurinol Induced DRESS Syndrome. J Assoc Physicians India 2014;62:63-5.

11. Zhang M, Solomon DH, Desai RJ, et al. Assessment of Cardiovascular Risk in Older Patients With Gout Initiating Febuxostat Versus Allopurinol: Population- 
Based Cohort Study. Circulation 2018;138:1116-26.

12. White WB, Saag KG, Becker MA, et al. Cardiovascular Safety of Febuxostat or Allopurinol in Patients with Gout. N Engl J Med 2018;378:1200-10.

13. Kang EH, Choi HK, Shin A, et al. Comparative cardiovascular risk of allopurinol versus febuxostat in patients with gout: a nation-wide cohort study. Rheumatology (Oxford) 2019;58:2122-9.

14. Zhang T, Pope JE. Cardiovascular effects of uratelowering therapies in patients with chronic gout: a systematic review and meta-analysis. Rheumatology (Oxford) 2017;56:1144-53.

15. Liu CW, Chang WC, Lee CC, et al. The net clinical benefits of febuxostat versus allopurinol in patients with gout or asymptomatic hyperuricemia - A systematic review and meta-analysis. Nutr Metab Cardiovasc Dis 2019;29:1011-22.

16. Becker MA, Schumacher HR Jr, Wortmann RL, et al. Febuxostat compared with allopurinol in patients with hyperuricemia and gout. N Engl J Med 2005;353:2450-61.

17. Schumacher HR Jr, Becker MA, Wortmann RL, et al. Effects of febuxostat versus allopurinol and placebo in reducing serum urate in subjects with hyperuricemia and gout: a 28-week, phase III, randomized, double-blind, parallel-group trial. Arthritis Rheum 2008;59:1540-8.

18. Becker MA, Schumacher HR, MacDonald PA, et al. Clinical efficacy and safety of successful longterm urate lowering with febuxostat or allopurinol in subjects with gout. J Rheumatol 2009;36:1273-82.

19. Becker MA, Schumacher HR, Espinoza LR, et al. The urate-lowering efficacy and safety of febuxostat in the treatment of the hyperuricemia of gout: the CONFIRMS trial. Arthritis Res Ther 2010;12:R63.

20. Kamatani N, Fujimori S, Hada T, et al. An allopurinolcontrolled, randomized, double-dummy, double-blind, parallel between-group, comparative study of febuxostat (TMX-67), a non-purine-selective inhibitor of xanthine oxidase, in patients with hyperuricemia including those with gout in Japan: phase 3 clinical study. J Clin Rheumatol 2011;17:S13-8.

21. Jackson RL, Hunt B, MacDonald PA. The efficacy and safety of febuxostat for urate lowering in gout patients $\geq 65$ years of age. BMC Geriatr 2012;12:11.

22. Tausche AK, Christoph M, Forkmann M, et al. As compared to allopurinol, urate-lowering therapy with febuxostat has superior effects on oxidative stress and pulse wave velocity in patients with severe chronic tophaceous gout. Rheumatol Int 2014;34:101-9.
23. Huang X, Du H, Gu J, et al. An allopurinol-controlled, multicenter, randomized, double-blind, parallel betweengroup, comparative study of febuxostat in Chinese patients with gout and hyperuricemia. Int J Rheum Dis 2014;17:679-86.

24. Xu S, Liu X, Ming J, et al. A phase 3, multicenter, randomized, allopurinol-controlled study assessing the safety and efficacy of oral febuxostat in Chinese gout patients with hyperuricemia. Int J Rheum Dis 2015;18:669-78.

25. Yu KH, Lai JH, Hsu PN, et al. Safety and efficacy of oral febuxostat for treatment of HLA-B*5801-negative gout: a randomized, open-label, multicentre, allopurinolcontrolled study. Scand J Rheumatol 2016;45:304-11.

26. Foody J, Turpin RS, Tidwell BA, et al. Major Cardiovascular Events in Patients with Gout and Associated Cardiovascular Disease or Heart Failure and Chronic Kidney Disease Initiating a Xanthine Oxidase Inhibitor. Am Health Drug Benefits 2017;10:393-401.

27. Su CY, Shen LJ, Hsieh SC, et al. Comparing Cardiovascular Safety of Febuxostat and Allopurinol in the Real World: A Population-Based Cohort Study. Mayo Clin Proc 2019;94:1147-57.

28. Chen CH, Chen CB, Chang CJ, et al. Hypersensitivity and Cardiovascular Risks Related to Allopurinol and Febuxostat Therapy in Asians: A Population-Based Cohort Study and Meta-Analysis. Clin Pharmacol Ther 2019;106:391-401.

29. Ju C, Lai RWC, Li KHC, et al. Comparative cardiovascular risk in users versus non-users of xanthine oxidase inhibitors and febuxostat versus allopurinol users. Rheumatology (Oxford) 2020;59:2340-9.

30. Nikolov NP, Seymour S, Neuner R, et al. Underscoring the Importance of Allopurinol in Treating Gout: Results of a Food and Drug Administration-Mandated Safety Trial. Arthritis Rheumatol 2020;72:877-8.

31. Rajendra NS, Ireland S, George J, et al. Mechanistic insights into the therapeutic use of high-dose allopurinol in angina pectoris. J Am Coll Cardiol 2011;58:820-8.

32. Gupta MK, Singh JA. Cardiovascular Disease in Gout and the Protective Effect of Treatments Including UrateLowering Therapy. Drugs 2019;79:531-41.

33. Wei L, Mackenzie IS, Chen Y, et al. Impact of allopurinol use on urate concentration and cardiovascular outcome. $\mathrm{Br}$ J Clin Pharmacol 2011;71:600-7.

34. Kelkar A, Kuo A, Frishman WH. Allopurinol as a cardiovascular drug. Cardiol Rev 2011;19:265-71.

35. Thanassoulis G, Brophy JM, Richard H, et al. Gout, 
allopurinol use, and heart failure outcomes. Arch Intern Med 2010;170:1358-64.

36. Kok VC, Horng JT, Chang WS, et al. Allopurinol therapy in gout patients does not associate with beneficial cardiovascular outcomes: a population-based matchedcohort study. PLoS One 2014;9:e99102.

37. Kim SC, Schneeweiss S, Choudhry N, et al. Effects of xanthine oxidase inhibitors on cardiovascular disease in patients with gout: a cohort study. Am J Med 2015;128:653.e7-653.e16.

38. Borghi C, Rosei EA, Bardin T, et al. Serum uric acid and the risk of cardiovascular and renal disease. J Hypertens 2015;33:1729-41; discussion 1741.

39. Borghi C, Rodriguez-Artalejo F, De Backer G, et al. Serum uric acid levels are associated with cardiovascular risk score: A post hoc analysis of the EURIKA study. Int J
Cardiol 2018;253:167-73.

40. Stamp LK, Chapman PT. Urate-lowering therapy: current options and future prospects for elderly patients with gout. Drugs Aging 2014;31:777-86.

41. Sezai A, Soma M, Nakata K, et al. Comparison of febuxostat and allopurinol for hyperuricemia in cardiac surgery patients with chronic kidney disease (NU-FLASH trial for CKD). J Cardiol 2015;66:298-303.

42. Faruque LI, Ehteshami-Afshar A, Wiebe N, et al. A systematic review and meta-analysis on the safety and efficacy of febuxostat versus allopurinol in chronic gout. Semin Arthritis Rheum 2013;43:367-75.

43. Hung SI, Chung WH, Liou LB, et al. HLA-B*5801 allele as a genetic marker for severe cutaneous adverse reactions caused by allopurinol. Proc Natl Acad Sci U S A 2005;102:4134-9.
Cite this article as: Wang M, Zhang Y, Zhang M, Li H, Wen C, Zhao T, Xie Z, Sun J. The major cardiovascular events of febuxostat versus allopurinol in treating gout or asymptomatic hyperuricemia: a systematic review and meta-analysis. Ann Palliat Med 2021;10(10):10327-10337. doi: 10.21037/apm-21-1564 


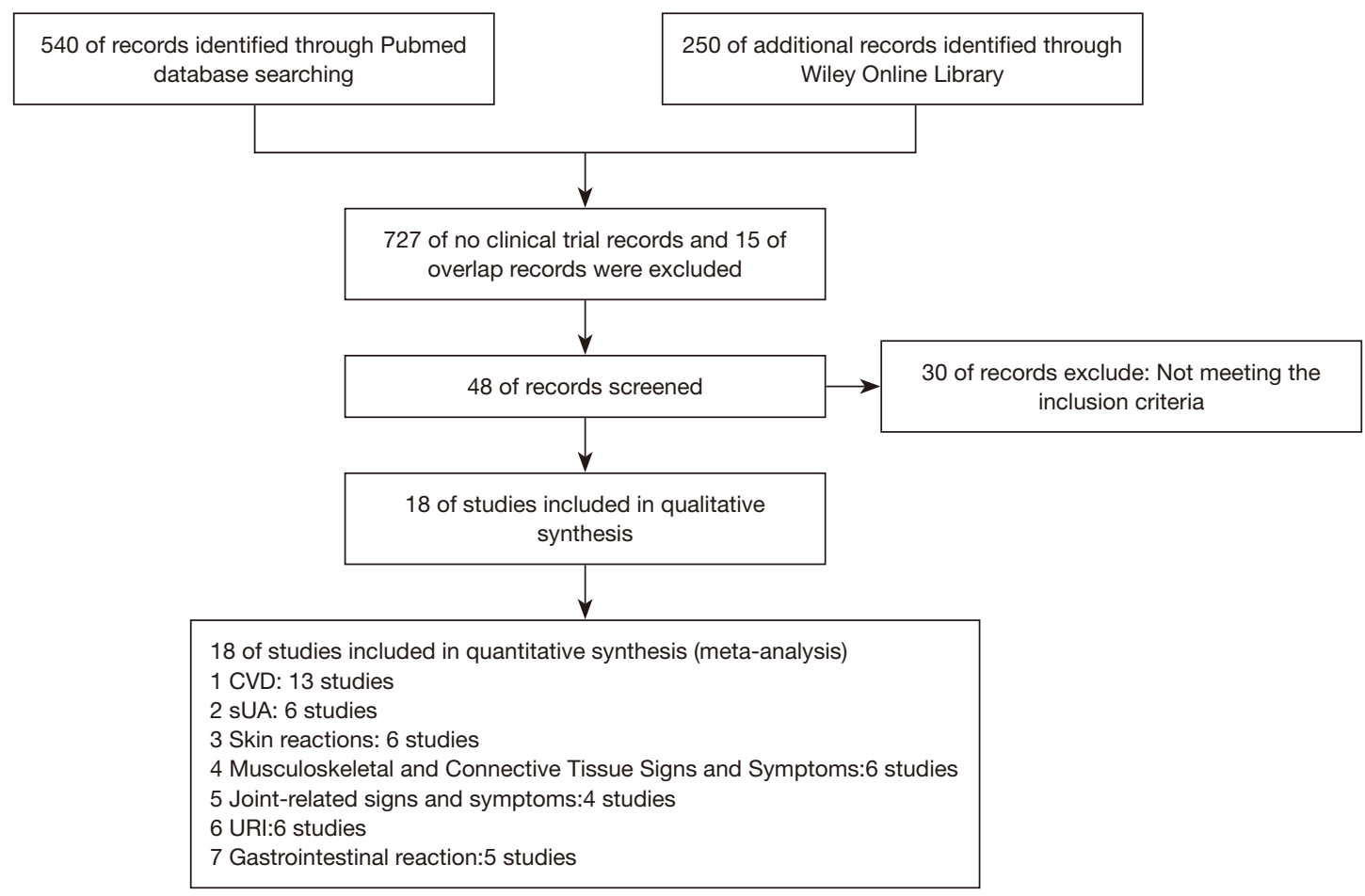

Figure S1 Flow diagram of study selection.

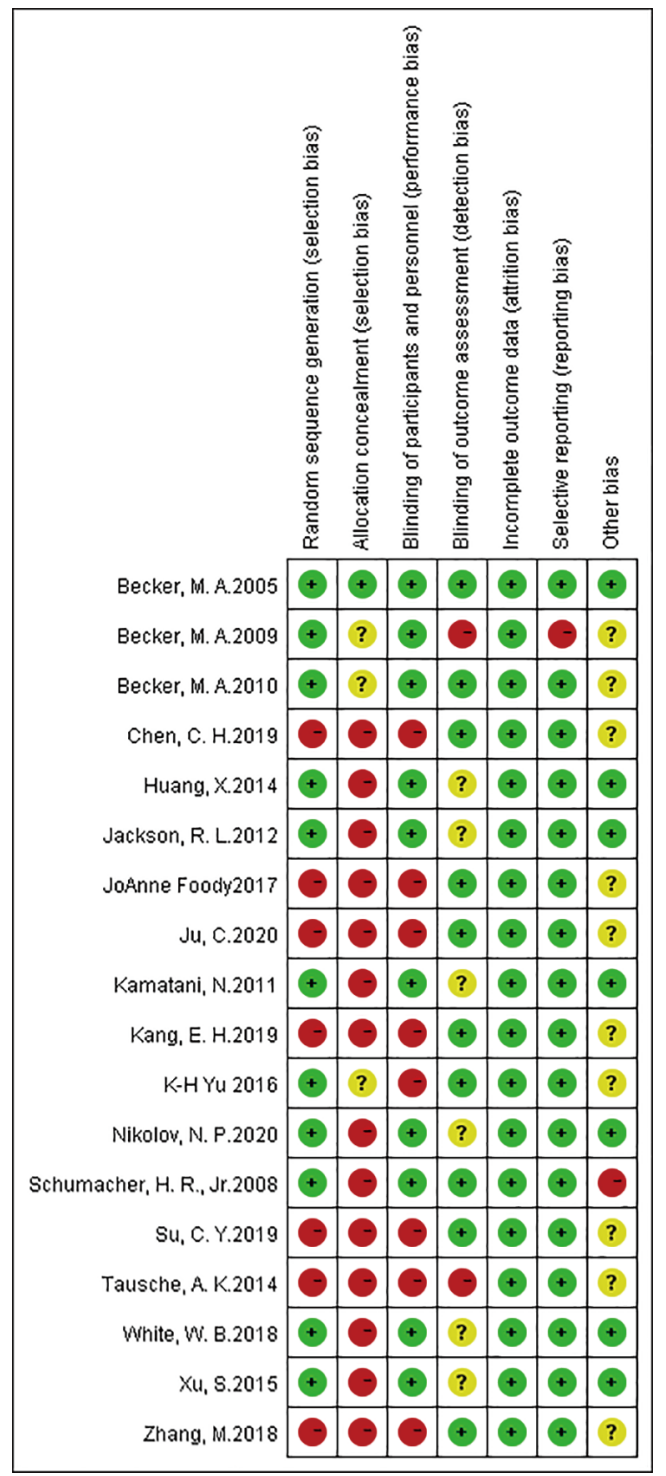

Figure S2 Risk of bias summary for uricosuric medications in randomized trials of gout or hyperuricemia. 
A

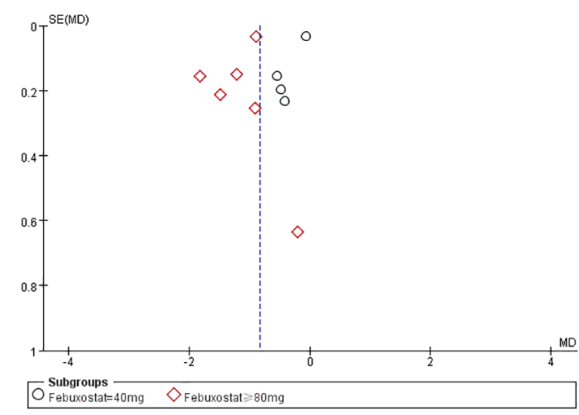

D

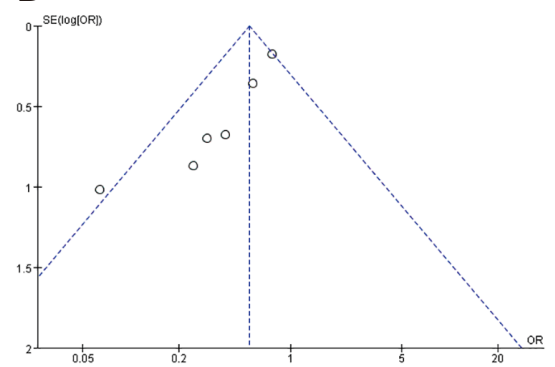

G

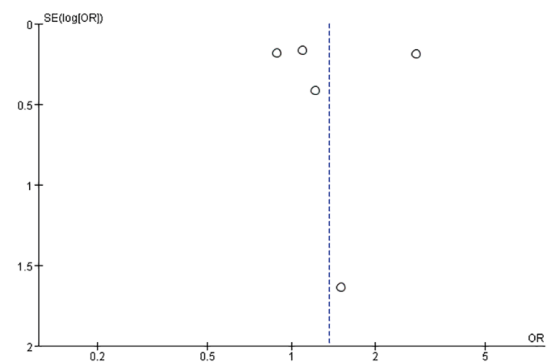

B

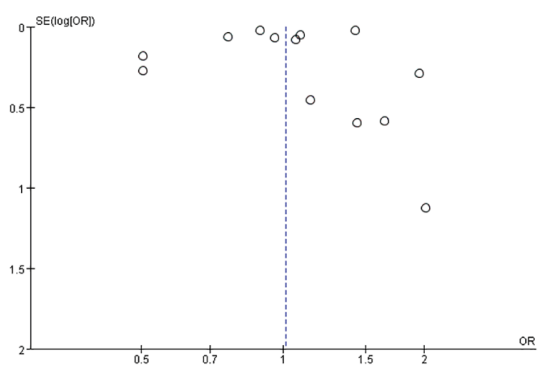

E

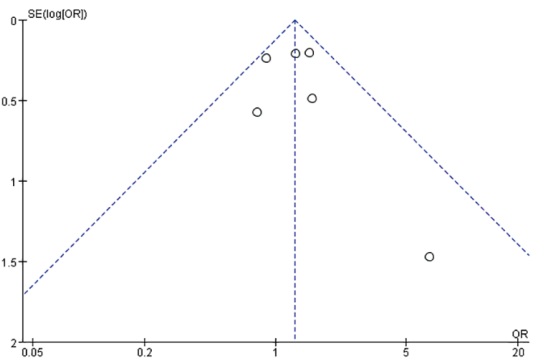

$\mathrm{H}$

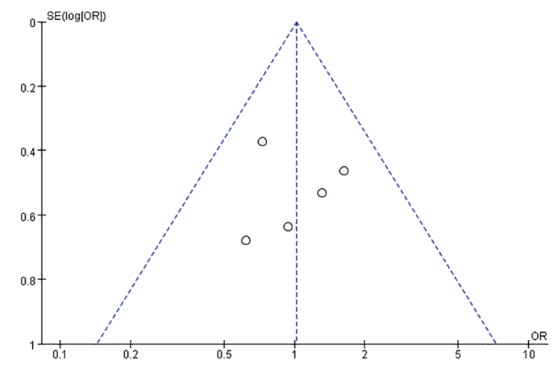

C

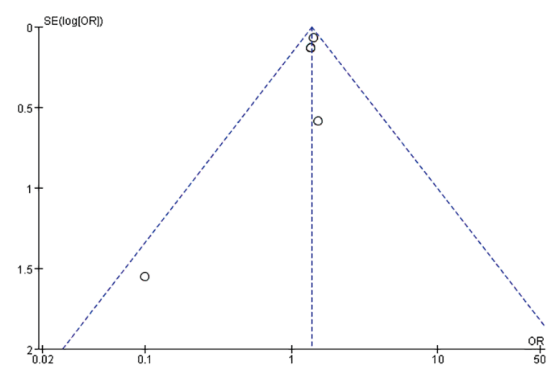

F

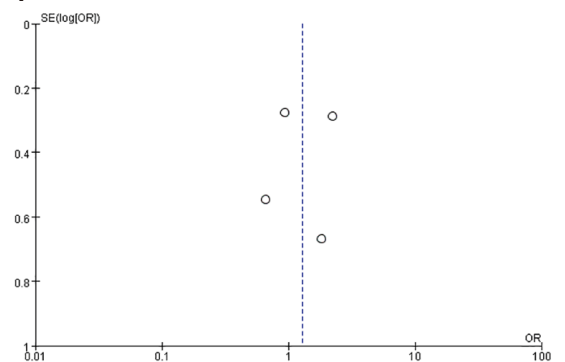

I

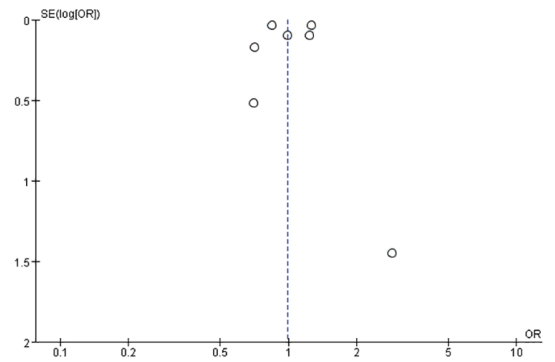

Figure S3 Funnel plots of the major cardiovascular (CV) events, serum uric acid (sUA) levels and the other adverse events comparing between febuxostat and allopurinol. A: sUA levels; B: major CV events; C: CV death, D: skin reactions; E: musculoskeletal and connective tissue signs and symptoms; F: joint-related signs and symptoms; G: upper respiratory infection (URI); H: gastrointestinal reaction, I: allcause mortality. 